\title{
Risk of acute brain lesions in dizzy patients presenting to the emergency room: who needs imaging and who does not?
}

\author{
Björn Machner ${ }^{1}\left[\right.$ [ J Jin Hee Choi $^{1} \cdot$ Peter Trillenberg ${ }^{1} \cdot$ Wolfgang Heide $^{2} \cdot$ Christoph Helmchen $^{1}$
}

Received: 26 March 2020 / Revised: 8 May 2020 / Accepted: 11 May 2020 / Published online: 27 May 2020

(c) The Author(s) 2020

\begin{abstract}
The usefulness of brain imaging studies in dizzy patients presenting to the emergency department (ED) is controversial. We aimed to assess the 'real-world' probability of ischemic stroke and other acute brain lesions (ABLs) in these patients to create an algorithm that helps decision-making on whether which and when brain imaging is needed. By reviewing medical records, we identified 610 patients presenting with dizziness, vertigo or imbalance to our university hospital's ED and receiving neurological workup. We collected timing/triggers of symptoms, $\mathrm{ABCD}^{2}$ score, focal neurological abnormalities, HINTS (head impulse, nystagmus, test-of-skew) and other central oculomotor signs. ABLs were extracted from CT/MRI reports. Uni-/multivariate logistic regression analyses investigated associations between clinical parameters and ABLs. Finally, the likelihood of ABLs was assessed for different clinically defined subgroups ('dizziness syndromes'). Early CT (day 1) was performed in 539 (88\%) and delayed MR imaging (median: day 4) in 299 (49\%) patients. ABLs (89\% ischemic stroke) were revealed in 75 (24\%) of 318 patients with adequate imaging (MRI or lesion-positive CT). The risk for ABLs increased with the presence of central oculomotor signs (odds ratio 2.8, 95\% confidence interval 1.5-5.2) or focal abnormalities (OR 3.3, 95\% CI 1.8-6.2). The likelihood of ABLs differed between dizziness syndromes, e.g., HINTS-negative acute vestibular syndrome: $0 \%$, acute imbalance syndrome with $\mathrm{ABCD}^{2}$-score $\geq 4: 50 \%$. We propose a clinical pathway, according to which patients with HINTS-negative acute vestibular syndrome should not receive brain imaging, whereas imaging is suggested in dizzy patients with acute imbalance, central oculomotor signs or focal abnormalities.
\end{abstract}

Keywords Stroke $\cdot$ Nystagmus $\cdot$ Vertigo $\cdot$ Dizziness $\cdot$ CT $\cdot$ MRI

\section{Introduction}

Dizziness is one of the most common presenting complaints in emergency departments (EDs) [17, 30]. Diagnosing patients with dizziness is challenging and ED physicians often request neurological consultation and brain imaging studies to differentiate non-vestibular medical causes (e.g., orthostatic dizziness) from peripheral (e.g., benign paroxysmal positional vertigo) or central vestibular disorders (e.g., brainstem/cerebellar stroke) [11,27]. The likelihood of detecting an acute brain lesion (ABL), for instance a stroke,

Björn Machner

Bjoern.Machner@neuro.uni-luebeck.de

1 Department of Neurology, University Hospital SchleswigHolstein, Campus Lübeck, Ratzeburger Allee 160, 23538 Lübeck, Germany

2 Department of Neurology, General Hospital Celle, Celle, Germany inflammatory lesion or tumor, varies greatly depending on the clinical preselection process of patients and the modality and timing of brain imaging studies. Thus, numbers range between 3-4\% in unselected cohorts of dizzy patients in the ED $[15,30], 27 \%$ in patients with an acute transient vestibular syndrome [3], and up to $75 \%$ in clinically preselected cohorts with an acute (persistent) vestibular syndrome and a high-risk vascular profile [14]. Notwithstanding, a stroke is still missed in about $35 \%$ of patients presenting with acute dizziness to the ED [15].

Brain imaging studies can help to differentiate peripheral vestibular and central causes of dizziness [13]. However, the sensitivity for detecting an ABL, particularly ischemic stroke, depends on the imaging modality and its timing [33, 34]. Computed tomography (CT) is usually performed in the emergency setting, due to its around-the-clock and on-site availability in most of the hospitals. However, the diagnostic yield of CT brain imaging in the evaluation of non-preselected dizzy patients in the ED is low ( 2\%) [21]. One reason is the 
CT's low sensitivity $(\sim 10 \%)$ for acute ischemic strokes [2], especially if they occur in the posterior cranial fossa [33]. In line with that, more CT imaging in the ED does not lead to an improvement in stroke diagnosis [20]. A negative CT result may even falsely reassure the ED physician of a peripheral cause instead of the actual central dizziness [10]. Although magnetic resonance imaging (MRI) has a higher sensitivity for acute ischemic strokes than CT [2], within the first $48 \mathrm{~h}$ diffusion-weighted MRI still can be false negative in about $50 \%$ of small ischemic strokes in the posterior fossa [34]. Furthermore, MRI is not as available (and feasible) as CT for dizzy patients in the ED and conducting MRIs in all dizzy patients presenting to the ED would unnecessarily burden many patients as well as the health-care system, as it exceeds the acceptable cost limits of most national economies [28, 33].

Hence, there is a clear need of a clinical preselection to identify those patients with a high pre-test probability for an ABL on imaging. However, the sensitivity and specificity of clinical tests that allow differentiation between peripheral and central causes of dizziness, such as the head impulse test [5, 31] or the HINTS triad (head impulse, nystagmus, test of skew) $[14,16]$, largely depend on the experience of the examiner [18]. On the other hand, 'rater-independent' risk scores like the $\mathrm{ABCD}^{2}$-score [25] or single risk factors such as high age [26] are less sensitive/specific [32] and they can only provide a rough estimation on the overall probability of a cerebrovascular cause, which may not be helpful in the individual case.

To specify, ED physicians and neurologists, who may not be neuro-otology experts, are confronted on a daily basis with the following questions [6]: Does this dizzy patient needs brain imaging or not? Is a CT sufficient, or is an MRI necessary? Is an immediate imaging study required? How do we deal with a negative result?

We aimed to develop a practical guide based on empirical evidence. By reviewing medical records of more than 600 patients presenting with dizziness, vertigo or imbalance to our university hospitals' ED, we analyzed the frequency of brain imaging studies and the occurrence of ABLs. Taking established diagnostic algorithms for dizzy ED patients into account $[8,37]$, we assessed the association of ABLs with specific clinical signs and 'dizziness syndromes'. Based on the results, we propose a clinical pathway that stratifies dizzy patients into subgroups of those who (i) do not need any brain imaging, (ii) do not need early CT but delayed MRI or (iii) require urgent $\mathrm{CT}$ and additional, delayed MRI when necessary.

\section{Methods}

\section{Study design and setting}

For this retrospective, single-center, observational study we reviewed the medical records of patients who presented to the emergency department (ED) of the University of Lübeck Medical Center (Lübeck, Germany). The ED at this tertiary care university hospital encounters about 42,000 patients per year. It is staffed 24/7 with resident and attending internal medicine physicians as well as one neurology resident. There is around-the-clock access to computed tomography (CT) in the ED, whereas MRIs are usually performed electively during daytime.

The study was approved by the Ethics Committee of the University of Lübeck (18-146A).

\section{Study population}

Using the hospital's medical controlling, we identified all adult ( $\geq 18$ years of age) patients who were (i) presenting to the ED between January 1, 2016 and December 31, 2018, (ii) seen by a neurology resident in the ED and (iii) finally admitted to one of the hospital's neurological wards (general neurological ward or stroke unit). From these preselected cases, we opened the electronic medical records written by the neurology resident in the ED and searched for any of the following presenting complaints: 'dizziness', 'vertigo' or 'imbalance'. By applying these criteria, we included patients with an acute and unclear type of dizziness who required admission to a neurological ward for further diagnostics and treatment. We thereby excluded patients with dizziness due to a clear medical cause and patients with an identified benign paroxysmal positional vertigo (BPPV) who could be successfully treated in the ED and directly discharged home.

For patients with multiple eligible ED visits during the study period, only the first visit was included.

\section{Baseline measurements}

We collected information on patients' characteristics (age, sex), comorbidities (arterial hypertension, diabetes mellitus, atrial fibrillation, coronary heart disease) and past medical history (prior stroke/TIA, vestibular disorders including Meniere's disease, vestibular migraine, vestibular neuritis, BPPV). We obtained specific information on the presenting complaint including its character (e.g., 'spinning' or 'swaying'), whether it was persistent (continuously present at the time of presentation in the ED) or transient/episodic, the type of onset (sudden, slowly progressing), the duration and if there were any triggers (e.g., head motion, changes in body 
position, locomotion) or associated symptoms (nausea/vomiting, headache, hearing disturbances, diplopia, visual field abnormality, speech difficulty, hemi-symptoms including sensory, motor or coordination abnormalities).

From the documentation of the neurological examination, we extracted information on focal abnormalities (aphasia, dysarthria, visual field defects, facial weakness, limb weakness, limb ataxia, sensory impairment) and the HINTSrelevant information including the bedside head impulse test (bHIT), spontaneous nystagmus and test of skew. The HINTS triad was rated as 'negative' if (i) the bHIT was abnormal (corrective saccade after horizontal head thrust), (ii) the nystagmus' fast phase did not change direction with gaze and (iii) there was no skew deviation. HINTS were rated as 'positive' if bHIT was documented as normal, the nystagmus' fast phase alternated with gaze or a skew deviation was observed. We also collected information on the following central oculomotor (OM) signs: horizontal/vertical gaze-evoked nystagmus, vertical or purely torsional spontaneous nystagmus, ophthalmoparesis and disrupted ('saccadic') smooth-pursuit eye movements.

The results of the Dix-Hallpike maneuver, if performed, were categorized into 'BPPV-typical nystagmus' in the plane of a specific semicircular canal on positional maneuvers, 'atypical nystagmus' or 'no nystagmus'.

Using the blood pressure (RR) documented at ED presentation and parameters mentioned above, we calculated the $\mathrm{ABCD}^{2}$ score (range $0-7$ ) for each patient: age 60 years or older $=1$; blood pressure $\geq 140 / 90=1$; clinical features (unilateral weakness $=2$, speech disturbance $=1$ ); duration of symptoms ( $<10 \mathrm{~min}=0,10-59 \mathrm{~min}=1, \geq 60 \mathrm{~min}=2)$; and diabetes $=1$ [12]. In case of missing information on a specific $\mathrm{ABCD}^{2}$ item, this was assigned a score of 1 [25].

\section{Outcome measures}

Brain imaging results (CT, MRI) were abstracted from the official neuroradiological reports. The primary outcome was an $\mathrm{ABL}$ on brain imaging including infarction, hemorrhage, intracerebral tumor and inflammatory lesions of the central nervous system (CNS). Residual vascular, post-traumatic or post-interventional defects as well as known and stable tumors (e.g., meningioma) were not attributed as ABL, because they could hardly account for the acute dizziness as a presenting complaint.

CT scans were performed on a 64-slice CT scanner (Siemens SOMATOM Definition AS+) during the patients' stay in the ED. MRI scans, which always included axial T2 fluid-attenuated inversion recovery (FLAIR) and diffusionweighted images (DWI) besides other sequences, were obtained on a 1.5 or 3.0 T Philips Achieva MRI scanner, usually some days after admission.

\section{Statistical analysis}

Statistical analyses were performed using SPSS 22.0 (IBM Corp., Somer/NY, US). Descriptive statistics were calculated for all variables of interest, and data are presented as counts and percentages.

Risk factors potentially associated with the primary outcome were assessed via univariate and, if significant, multivariate logistic regression analysis (LRA). Results from the LRA are given as odds ratios (ORs) with $95 \%$ confidence interval. $P$ values $<0.05$ were considered significant.

The frequency of ABLs was calculated separately for the two imaging modalities and for different clinical subgroups of patients. The patient's assignment to a distinct subgroup was based on recently suggested clinical algorithms for dizzy patients in the ED $[8,37]$. The algorithms use information from the patient's history (timing/trigger of symptoms) and targeted clinical examination (oculomotor signs) to stratify the patient to one specific 'dizziness syndrome'. These syndromes encompassed the 'spontaneous transient vestibular syndrome' (sTVS; vestibular symptoms $<24 \mathrm{~h}$ and not present at presentation in the ED, no trigger in the history), 'triggerable episodic vestibular syndrome' (tEVS; episodic vestibular symptoms that are provoked by specific triggers, e.g., postural change like in BPPV), 'acute vestibular syndrome' (AVS; vestibular symptoms and spontaneous nystagmus, continuously present at ED presentation), and 'acute imbalance syndrome' (AIS; acute onset of an unsteadiness in stance and gait, still persistent at ED presentation, no spontaneous nystagmus). Based on the findings from the clinical examination, tEVS patients were further assigned to either a BPPV cohort (typical nystagmus on Dix-Hallpike) or a central positional vertigo (CPV) cohort (atypical or no nystagmus on Dix-Hallpike). Likewise, AVS patients were divided into a HINTS-positive 'central' group and a HINTS-negative 'peripheral' group. Finally, using the $\mathrm{ABCD}^{2}$-score, patients with an AIS were split into a highrisk ( $\geq 4$ points) and a low-risk ( $<4$ points) AIS subgroup.

\section{Results}

\section{Demographic, clinical and imaging characteristics}

610 patients fulfilled the eligibility criteria and were included in the final analysis. 539 of these patients (88.4\%) received a CT in the ED. An MRI was conducted in 299 patients (49.0\% of all patients); this usually happened with a delay (median: 4 days) but always within 18 days after admission. Most of the patients with an MRI first received a CT $(n=279)$, i.e., only 20 patients received an MRI without having an initial CT. Only few patients received an MRI on the first day $(n=9,3.0 \%)$. 
Table 1 provides the demographic and clinical characteristics of the patients, separately for the whole study group and different subgroups (patients with ABLs, patients without ABLs, patients without any imaging).

ABLs were identified in 36 of 557 patients (5.6\%) receiving early CT and in 56 of 299 patients (18.1\%) who received MRI. From the 56 patients with an ABL on MRI, $n=17$ already had the lesion revealed on the initial CT, $n=37$ had a normal CT result and $n=2$ did not have a CT in the ED. There were 19 patients with ABLs detected by the initial CT who did not receive additional MRI. Overall, 75 (23.6\%) of 318 dizzy patients who received adequately sensitive brain imaging eventually had an ABL.

The ABLs' most common etiology was ischemic stroke $(n=67,89.3 \%)$, besides two patients with an intracerebral hemorrhage (2.7\%), three patients with inflammatory CNS lesions $(4.0 \%)$, two patients with an intracerebral tumor $(2.7 \%)$ and one patient with a post-epileptic edema of the hippocampal region.

Patients who did not receive any brain imaging (Table 1, last column) were mostly female (75\%) and often reported transient dizziness symptoms (49\%) and positional changes as trigger (31\%). Many of them had a previous history of vestibular disorders (35\%) and only few exhibited central OM signs (8\%) or focal abnormalities (6\%) on clinical examination.

\section{Clinical predictors for ABLs}

To identify clinical markers predicting an 'ABL' (dependent variable), odds ratios were calculated for several clinical parameters by using univariate/multivariate logistic regression analyses (Table 2).

Univariate analyses revealed that ABLs were more likely in patients with (i) preexisting arterial hypertension, (ii) high-risk $\mathrm{ABCD}^{2}$ score $\geq 4$, (iii) any central $\mathrm{OM}$ sign and (iv) any focal abnormality on clinical examination. In contrast, ABLs were found to be less likely in (v) patients who reported 'vertigo with a sense of spinning' as the dizziness' character and (vi) those with transient dizziness symptoms.

In the multivariate model, only central OM signs (OR 2.8, 95\% CI 1.5-5.2) and focal abnormalities on clinical examination (OR 3.3, 95\% CI 1.8-6.2) remained statistically significantly positive predictors for ABLs, while the transient

Table 1 Characteristics of the whole study population and different subgroups of patients with respect to the results of the brain imaging studies

\begin{tabular}{|c|c|c|c|c|}
\hline Characteristics & All patients $(n=610)$ & $\begin{array}{l}\text { Patients with ABL on } \\
\text { CT or MRI }(n=75)\end{array}$ & $\begin{array}{l}\text { Patients with no ABL } \\
\text { on MRI }(n=243)\end{array}$ & $\begin{array}{l}\text { Patients without } \\
\text { brain imaging } \\
(n=51)\end{array}$ \\
\hline Age [years; mean \pm SD (median)] & $65 \pm 16(67)$ & $66 \pm 14(66)$ & $64 \pm 15(64)$ & $61 \pm 19(61)$ \\
\hline Female & $319(52)$ & $34(45)$ & $129(53)$ & $38(75)$ \\
\hline \multicolumn{5}{|l|}{ Comorbidities/vascular risk factors } \\
\hline Diabetes & $85(14)$ & $15(20)$ & $32(13)$ & $2(4)$ \\
\hline Hypertension & $318(52)$ & $49(65)$ & $121(50)$ & $20(39)$ \\
\hline Prior stroke & $80(13)$ & $9(12)$ & $33(14)$ & $3(6)$ \\
\hline $\mathrm{ABCD}^{2}$-score $[$ mean $\pm \mathrm{SD}($ median $)]$ & $2.9 \pm 0.9(3.0)$ & $3.3 \pm 1.2(3.0)$ & $2.9 \pm 0.9(3.0)$ & $2.7 \pm 0.8(3.0)$ \\
\hline $\mathrm{ABCD}^{2} \geq 4$ (high risk) & $121(20)$ & $27(36)$ & $42(17)$ & $9(18)$ \\
\hline Previous diagnosis of a vestibular disorder & $88(14)$ & $5(7)$ & $38(16)$ & $18(35)$ \\
\hline \multicolumn{5}{|l|}{ Targeted history of the symptom 'dizziness' } \\
\hline Vertigo ('spinning') & $301(49)$ & $25(33)$ & $115(47)$ & $33(65)$ \\
\hline Sudden onset & $404(66)$ & $48(64)$ & $161(66)$ & $32(63)$ \\
\hline Episodic & $170(28)$ & $6(8)$ & $70(29)$ & $25(49)$ \\
\hline Triggerable & $69(11)$ & $2(3)$ & $23(10)$ & $16(31)$ \\
\hline Positional & $61(10)$ & $2(3)$ & $18(7)$ & $16(31)$ \\
\hline Associated CNS symptoms & $125(21)$ & $36(48)$ & $46(19)$ & $2(4)$ \\
\hline Headache & $70(12)$ & $9(12)$ & $32(13)$ & $2(4)$ \\
\hline Hearing disturbance, tinnitus & $55(9)$ & $4(5)$ & $26(11)$ & $5(10)$ \\
\hline \multicolumn{5}{|l|}{ Findings on clinical examination } \\
\hline Any central oculomotor sign & $124(20)$ & $33(44)$ & $41(17)$ & $4(8)$ \\
\hline Any focal abnormality & $136(22)$ & $41(55)$ & $51(21)$ & $3(6)$ \\
\hline Initially admitted to the stroke unit & $344(56)$ & $64(85)$ & $148(61)$ & $0(0)$ \\
\hline
\end{tabular}

Data are $n(\%)$ unless otherwise indicated

$A B L$ acute brain lesion 
Table 2 Clinical predictors for an acute brain lesion in 318 patients presenting with dizziness to the emergency department

\begin{tabular}{|c|c|c|c|c|}
\hline \multirow[t]{2}{*}{ Parameters } & \multicolumn{2}{|c|}{ Univariate analysis } & \multicolumn{2}{|c|}{ Multivariate analysis } \\
\hline & OR $(95 \% \mathrm{CI})$ & $p$ value & OR (95\% CI) & $p$ value \\
\hline \multicolumn{5}{|l|}{ Vascular risk profile } \\
\hline Age $\geq 60$ years & $1.2(0.7-2.0)$ & 0.603 & & \\
\hline Arterial hypertension & $1.9(1.1-3.3)$ & $\mathbf{0 . 0 1 9}$ & $1.6(0.9-3.0)$ & 0.103 \\
\hline Diabetes & $1.7(0.9-3.3)$ & 0.148 & & \\
\hline Prior stroke & $0.9(0.4-1.9)$ & 0.724 & & \\
\hline $\mathrm{ABCD}^{2}$-score $\geq 4$ ('high risk') & $2.7(1.5-4.8)$ & 0.001 & $1.5(0.8-3.0)$ & 0.245 \\
\hline \multicolumn{5}{|l|}{ Symptomatology } \\
\hline Vertigo ('spinning') & $0.6(0.3-0.9)$ & $\mathbf{0 . 0 3 4}$ & $0.6(0.3-1.1)$ & 0.127 \\
\hline Sudden onset & $0.9(0.5-1.6)$ & 0.719 & & \\
\hline Transient symptoms & $0.2(0.1-0.5)$ & 0.001 & $0.3(0.1-0.6)$ & 0.004 \\
\hline Positional change as trigger & $0.3(0.1-1.5)$ & 0.157 & & \\
\hline Hearing disturbance & $0.5(0.2-1.4)$ & 0.173 & & \\
\hline Headache & $0.9(0.4-2.0)$ & 0.792 & & \\
\hline Associated CNS symptoms & $1.0(1.0-1.0)$ & 0.058 & & \\
\hline \multicolumn{5}{|l|}{ Clinical examination } \\
\hline Any central oculomotor sign & $3.9(2.2-6.8)$ & $<0.001$ & $2.8(1.5-5.2)$ & 0.001 \\
\hline Any focal abnormality & $4.5(2.6-7.9)$ & $<0.001$ & $3.3(1.8-6.2)$ & $<0.001$ \\
\hline
\end{tabular}

Only patients with sufficiently sensitive brain imaging (MRI or lesion-positive CT) were included in this analysis, which applied to 318 of 610 patients nature of symptoms was associated with a reduced risk for ABLs (OR 0.3, 95\% CI 0.1-0.6).

\section{Occurrence of ABLs within clinically defined patient subgroups (dizziness syndromes)}

We analyzed the frequency of patients who received brain imaging and the number of cases in which an ABL was detected, separately for the two imaging modalities and the different clinically defined subgroups (see "Methods"). The probability of detecting a lesion was greater with MR (Fig. 1b) than CT imaging (Fig. 1a), and more importantly, the clinical subgroups were associated with a very different risk of ABLs.

There were 'zero-risk' subgroups including patients with episodic vertigo triggered by positional change and typical nystagmus on Dix-Hallpike (BPPV group), whereas similarly presenting patients with no or an atypical nystagmus, indicating central positional vertigo (CPV group), revealed ABLs in $6 \%$ of CT and $11 \%$ of MRI scans.

Not a single patient with an acute vestibular syndrome (AVS), in whom the complete HINTS triad was negative, revealed ABLs. MRIs disclosed ABLs in $11 \%$ of those AVS patients with an abnormal bHIT, but without the other two HINTS items (Fig. 1b).

The ABL risk was highest in patients with an 'acute imbalance syndrome (AIS)', defined by persistent dizziness and imbalance but no nystagmus. The likelihood of detecting an $\mathrm{ABL}$ was $11 \%$ on the initial $\mathrm{CT}$, increasing to $33 \%$ on the delayed MRI. AIS patients with a high-risk $\mathrm{ABCD}^{2}$-score $\geq 4$ even revealed ABLs in $50 \%$ of MRIs (Fig. 1b).

The probability of detecting an ABL in patients with 'spontaneous transient vestibular syndrome (sTVS)' was very low on early CT imaging (2\%) and only moderate on delayed MRI (8\%). Separate analysis of high-risk sTVS patients with an $\mathrm{ABCD}^{2}$-score $\geq 4$ and/or focal CNS symptoms during the attack $(n=31)$ did not increase the specificity, i.e., their probability of ABLs on MRI $(2 / 20,10 \%)$ was almost as high as in the whole sTVS group. Accordingly, two patients with a low-risk $\mathrm{ABCD}^{2}$-score $<4$ and no history of focal CNS symptoms still revealed ABLs (2/31, 6.5\%).

\section{Discussion}

We analyzed the 'real-world' frequency and diagnostic yield of brain imaging studies (CT, MRI) in patients presenting with dizziness, vertigo or imbalance to the ED, excluding those with a clear medical cause or uncomplicated BPPV. Early CT imaging was conducted in over $80 \%$ of the patients and almost every second patient later received an MRI (median 4 days). Dizzy patients who did not receive brain imaging were mostly female, typically reported transient/ triggerable symptoms and seldom exhibited central oculomotor signs or other focal abnormalities on clinical examination. This most probably reflects a preexisting selection strategy in our ED to especially prevent young women with 


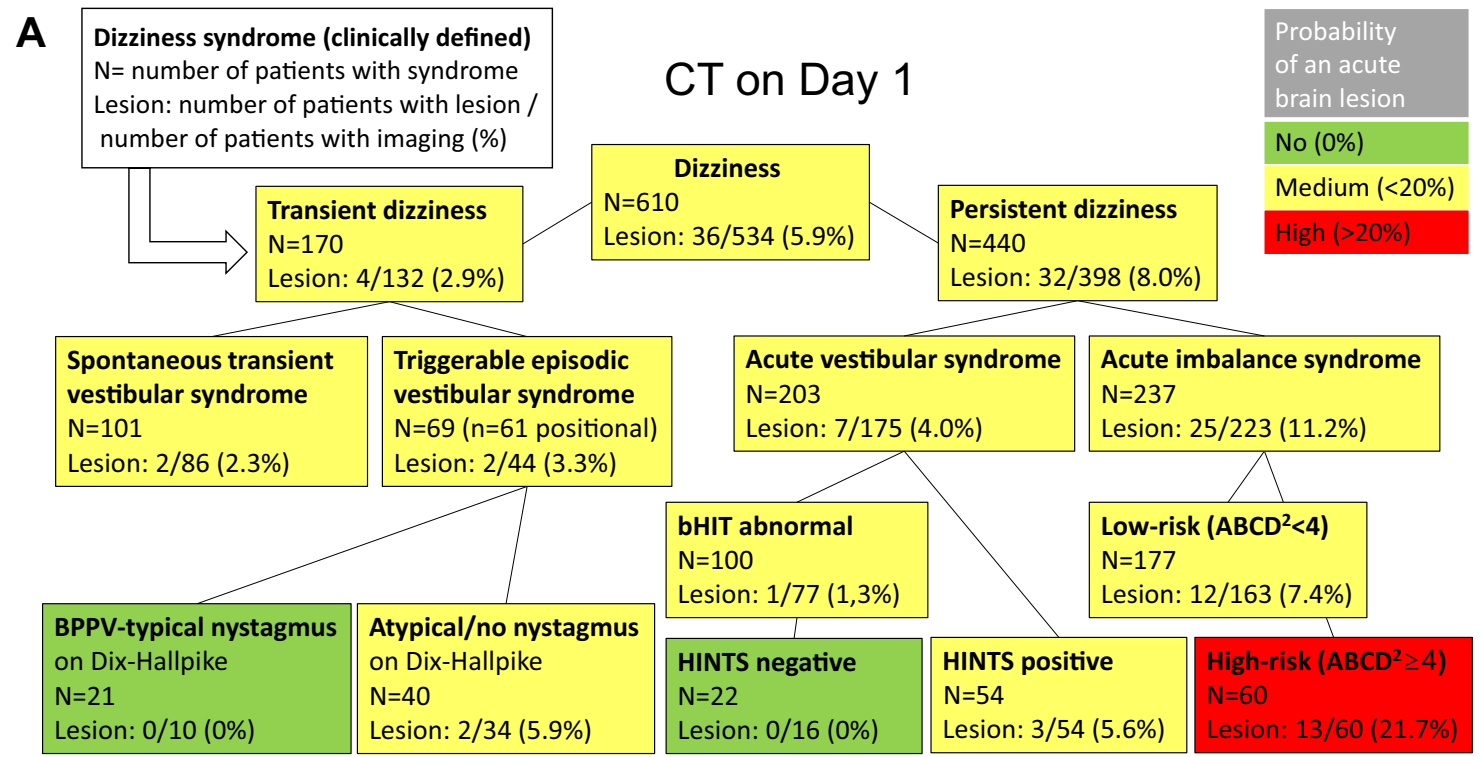

B

\section{MRI on Day 4}

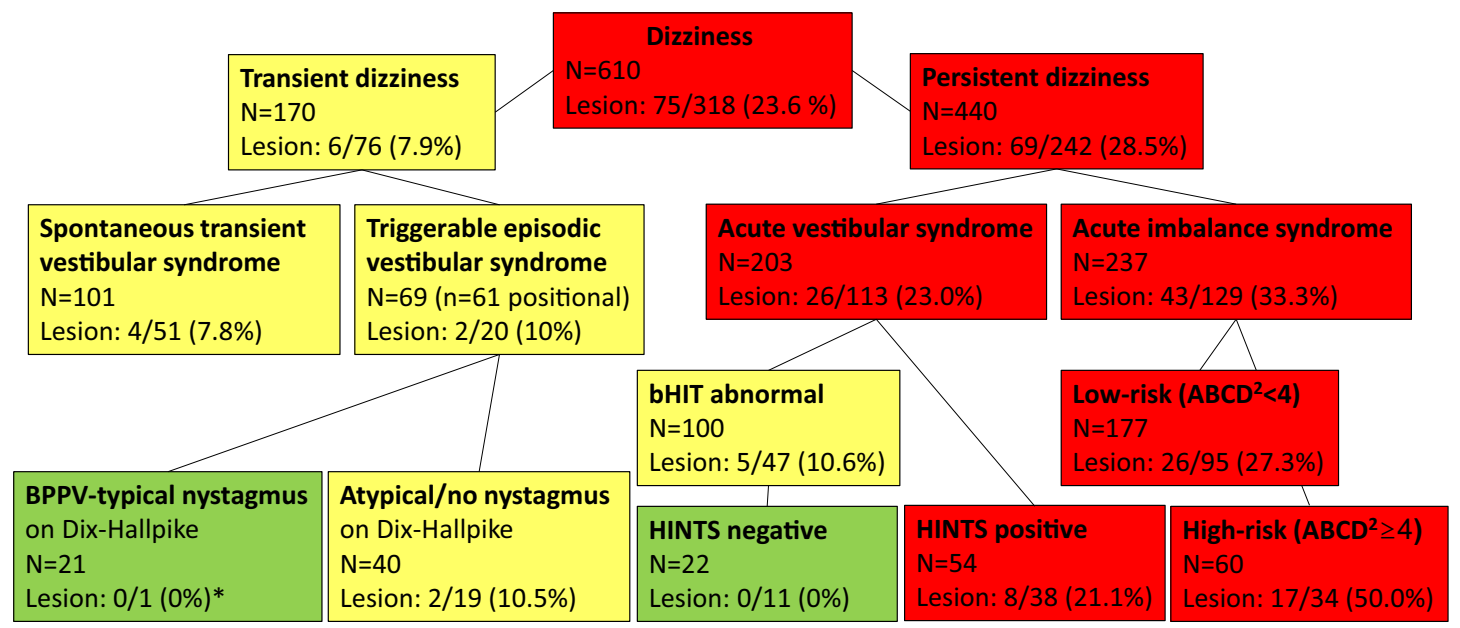

Fig. 1 Probability of an acute brain lesion as detected by early CT a or delayed MR imaging $\mathbf{b}$ in dependence of the patients' clinical subspecification ('dizziness syndrome'). For the purpose of clarity, we color coded the probability/risk to have an acute brain lesion (ABL) revealed by the respective imaging study (green: no risk, yellow: low-medium risk, red: high risk). a Early CT imaging performed in 534 of 610 'dizzy' patients revealed ABLs in 36 of them (5.9\%). Further stratification of the patients by using information from targeted history taking and clinical examination can increase the probability of detecting ABLs on CT to over $20 \%$ (e.g., 'acute imbalance syn-

transient symptoms and normal neurological examination from unnecessary CT imaging.

Every fourth patient (24\%) revealed an ABL (usually ischemic stroke) on adequately sensitive brain imaging. This could be taken as an argument to perform brain imaging in every dizzy patient presenting to the ED. However, in light of the high economic burden and overexposure of patients drome (AIS) with high-risk $\mathrm{ABCD}^{2}$-score'). b Delayed MRI is more sensitive in detecting ABLs and identifies high-risk subgroups (e.g., $\mathrm{ABLs}$ in $50 \%$ of AIS patients with $\mathrm{ABCD}^{2} \geq 4$ ), but also no-risk subgroups (e.g., 0\% ABLs in HINTS-negative AVS patients). Notably, b also includes those patients with an ABL already detected on the early CT ('lesion-positive' CT) who did not receive a redundant MRI. *Patients with benign paroxysmal positional vertigo (BPPV) were generally rare in our study cohort as they were usually identified and directly discharged from the ED

to imaging studies, we should make use of clinical markers that allow to preselect those dizzy patients who have a high pre-test probability for a central lesion.

In our cohort of dizzy ED patients, we found two clinical markers to be independent predictors for an ABL: any central oculomotor sign or a focal abnormality detected on neurological examination. In contrast, vascular risk factors 
such as arterial hypertension or an $\mathrm{ABCD}^{2}$-score $\geq 4$ were only associated with ABLs in the univariate, but not in the multivariate logistic regression analysis. These results are in line with the previously proposed markers for central causes of acute dizziness: Navi and colleagues identified 'focal examination abnormality', 'higher age ( $\geq 60$ years)' and 'imbalance as presenting complaint' as independent predictors [26]. Kerber et al. found the 'continuous $\mathrm{ABCD}^{2}$ score', a 'central pattern on the oculomotor assessment', 'any other CNS feature' on clinical examination and 'prior stroke' to be related with stroke in patients with 'new and continuous dizziness' [16]. Taken together, brain imaging is strongly suggested in dizzy patients who exhibit any central oculomotor sign or focal abnormality on neurological examination. In contrast, vascular risk factors such as higher age or an $\mathrm{ABCD}^{2}$-score $\geq 4$ are not consistently found to be reliable and independent predictors for ABLs in dizzy patients and should therefore not solely trigger imaging studies.

But how shall we manage dizzy patients in the ED who do not reveal any central oculomotor sign or focal abnormality? Conducting brain imaging in those with at least one vascular risk factor would lead to an excessive use of imaging studies in many dizzy patients with an actually benign peripheral-vestibular or non-vestibular cause. On the other hand, applying risk factors as 'conditio sine qua non' may prevent necessary imaging studies in juvenile stroke patients with a dissection or thromboembolic occlusion of the vertebral artery [19].

We rather suggest a clinical stratification of patients into specific 'dizziness syndromes', based on symptoms (timing, triggers) and signs from a targeted clinical examination $[8,37]$. We could show that these previously proposed (and slightly modified) algorithms can be successfully applied to clinical routine in the ED when dizzy patients are assessed by non-experts in neuro-otology. Using their clinical assessment allowed to distinguish dizzy patients with a zero, moderate or high pre-test probability for ABLs on brain imaging. Based on that, we developed a clinical pathway (Fig. 2) that can be used practically by clinicians working in EDs to decide whether an individual dizzy patient should receive brain imaging or not.

To specify, patients with an episodic vestibular syndrome triggered by positional change should only receive brain imaging when there is no BPPV-typical nystagmus on Dix-Hallpike maneuver and CPV is suspected. In AVS patients, brain imaging is dispensable if the HINTS-triad is negative. While the HINTS' high sensitivity for detecting strokes in dizzy patients is known to neuro-oto-/ophthalmological experts [14, 32], our study impressively confirms their usefulness when applied by neurology residents under 'real-world' conditions in the ED. When the bHIT is used as a single test to differentiate peripheral and central vestibular

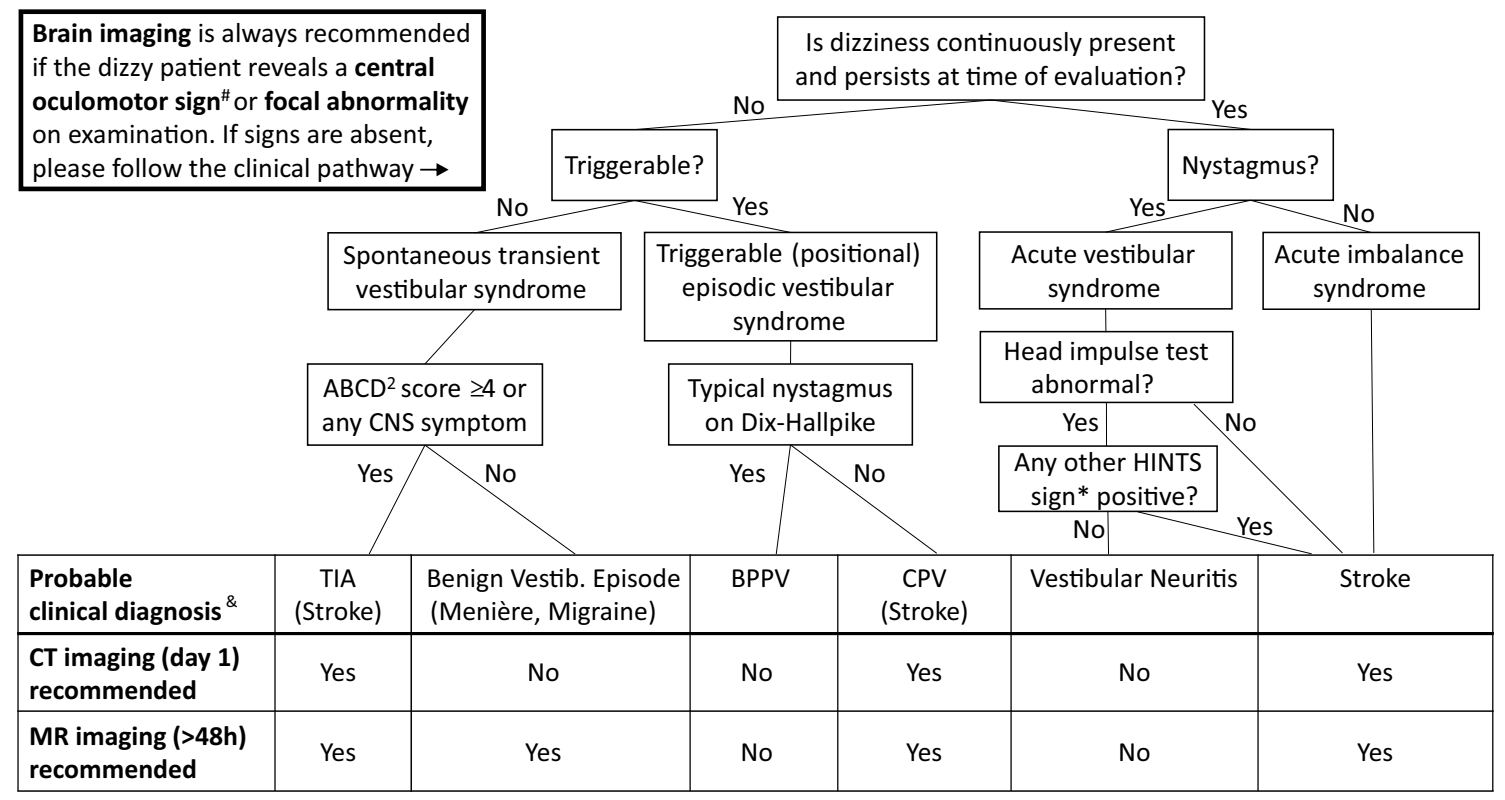

Fig. 2 Clinical pathway to help decision-making on brain imaging in patients presenting to the ED with dizziness, vertigo or imbalance and without a general medical cause ${ }^{+}$. TIA transient ischemic attack, $B P P V$ benign paroxysmal positional vertigo, $C P V$ central positional vertigo. ${ }^{+}$General medical causes comprise various toxic, metabolic, infectious, or cardiovascular diseases (see Edlow et al. 2018 [8]). ${ }^{\#}$ Central oculomotor signs include: vertical or purely torsional spon- taneous nystagmus, horizontal/vertical gaze-evoked nystagmus, gaze palsies, bilaterally disrupted smooth pursuit eye movements. ${ }^{*}$ HINTS are positive ('central') if any of the following signs is present: normal head impulse test, the nystagmus' fast phase alternating with gaze, skew deviation with a refixation on cover test. ${ }^{\S}$ The MRI may be dispensable if the lesion has already been detected by early CT. ${ }^{\&}$ Only the most likely and most relevant differential diagnosis is stated. 
causes in AVS, neurology residents even revealed a higher sensitivity for central pathologies than experts in previous studies: only $11 \%$ of patients with an abnormal bHIT (indicating peripheral-vestibular pathology) later disclosed strokes on MRI versus $30 \%$ of patients in studies using experts' assessments [5, 31]. We like to emphasize, however, that we definitely recommend using the complete HINTS triad, or even the HINTS+ assessment (including examination of a sudden hearing loss) [32], because the bHIT as a single test may be abnormal (and thereby falsely indicate an isolated peripheral vestibulopathy) in some patients with circumscribed ponto-cerebellar lesions or a combined peripheral-central vestibulopathy as in AICA infarctions that include the labyrinthine artery [4, 22, 31]. AVS patients with a normal bHIT or any other positive HINTS sign (skew deviation, nystagmus' fast phase alternating, sudden hearing loss) should always receive brain imaging as previously suggested $[14,32]$ and confirmed in our cohort (21\% ABLs).

Patients with acute imbalance exhibited the highest risk for ABLs among all dizziness syndromes (ABLs in 50\% of vascular high-risk AIS patients). Even AIS patients with a 'low risk' $\mathrm{ABCD}^{2}$-score $<4$ had a considerable risk of ABLs (27\%). Therefore, we would extend the recommendation by Zwergal et al. [37] that brain imaging should be performed in all AIS patients independent of their individual vascular risk profile.

Patients with spontaneous transient vestibular syndrome (sTVS) remain the most challenging subgroup. Differential diagnoses encompass vertebrobasilar transient ischemic attacks (TIA), (first) manifestation of a 'benign' episodic vestibular disease (Meniere's disease, vestibular migraine) and also non-vestibular causes such as transient disturbances of blood glucose/electrolytes, cardiovascular disorders (arrhythmia) and attacks of phobic/functional vertigo [29]. Clinical examination can hardly contribute to making the diagnosis, as it is usually normal when the asymptomatic patient is assessed in the $\mathrm{ED}$, and transient (vestibular) symptoms are a proven risk factor for missing a stroke in the ED [36]. Brain imaging may be helpful, since diffusion-weighted MRI has been shown to detect acute ischemic lesions in 34\% of TIA patients [1] and in $15 \%$ of sTVS patients [3]. In our cohort, only $8 \%$ of sTVS patients revealed an ABL on MRI. However, absence of an MRI-DWI lesion does not rule out a transient ischemia [3]. Although we could not relevantly increase the probability of detecting ABLs when including the $\mathrm{ABCD}^{2}$-score and CNS symptoms during the attack into our model, as a practical approach we suggest to consider brain imaging in those sTVS patients with an $\mathrm{ABCD}^{2}$-score $\geq 4$ or accessory CNS symptoms. If any of these features is present, early brain imaging is recommended and, in case of sudden head or neck pain potentially indicating vascular dissection, an additional angiogram [9]. If markers are negative and this was the first vestibular episode, we would refrain from early CT but recommend a delayed MRI during the stay in the hospital. In case of recurrent episodes (most probable differential diagnoses: vestibular migraine, Meniere's disease), a non-urgent MRI should be performed once to exclude rare structural pathologies such as vestibular schwannoma [23] or a pathological contact between the eighth nerve and a cerebellar artery as in vestibular paroxysmia [35].

\section{Which imaging modality (CT or MRI) and when?}

In an ideal world, dizzy patients with clinical indicators of a central cause would primarily receive MRI because it has less radiation risks than $\mathrm{CT}$ and higher sensitivity for ischemic stroke and other CNS pathologies [13, 29]. Accordingly, in our study, an ABL was revealed in only 5.6\% of the patients who received an (early) CT versus $18.1 \%$ of patients who received a (delayed) MRI. Of course, this difference is also influenced by the temporal order effect (first $\mathrm{CT}$, second MRI), as the likelihood to detect an acute stroke lesion is generally greater $>48 \mathrm{~h}$ after symptom onset than on the first day.

However, most of the hospitals (even at tertiary medical centers in highly developed countries) have to deal with infrastructural restrictions such as a reduced number of available MRI slots and gaps in the around-the-clock availability of MRI-experienced staff. Moreover, MRI in the acute stage of vestibular disorders may sometimes not be feasible if the patient suffers from severe nausea and vomiting. Furthermore, there are time-critical situations where the imaging study must be conducted immediately, e.g., before thrombolysis in acute ischemic strokes. We therefore regard it more realistic to (i) preselect patients who require brain imaging according to our proposed clinical pathway, (ii) perform an early $\mathrm{CT}$ in the ED and (iii) add a delayed MRI when necessary. Performing the MRI with a temporal delay also reduces the risk of missing small vertebrobasilar infarcts due to false-negative DWI scans in the first $48 \mathrm{~h}$ [14, 34]. Another advantage is that the $\mathrm{CT}$ is sensitive enough to rule out an intracranial hemorrhage and that patients with suggested ischemic stroke can immediately receive antiplatelet medication (or anticoagulants under specific conditions) before the MRI is conducted 3 days later.

Notably, a lack of an ABL even on delayed MRI does not completely rule out a central etiology of the dizziness. First, there is the possibility of small MRI-negative brainstem strokes, which were estimated to have a prevalence between $6.8 \%$ [7] and up to 29\% [24]. Second, the CNS disorder may have been transient (e.g., TIA or vestibular migraine) and did not cause an ABL. On the other hand, signs or symptoms that were clinically suspected to be due to a central etiology may not always indicate true and acute CNS lesions, e.g., a mild dysmetria in elderly patients interpreted as limb ataxia 
or sensory symptoms during the vestibular attack which may also be due to a functional/phobic etiology.

\section{Limitations}

In contrast to a prospective controlled study, this study has some limitations that are implicitly due to its retrospective design and the data acquisition in a real-world setting. First, there may have been a selection bias during the initial assessment in the ED when deciding which patient needs to be admitted to the neurological service and which patient should receive brain imaging. Indeed, we cannot fully exclude that patients who did not receive any brain imaging or only a CT scan would have shown an acute brain lesion on proper MR imaging. Furthermore, patients were examined by different neurology residents with different levels of experience and they all followed a certain clinical routine but they did not fill out a structured study protocol, e.g., when assessing the HINTS and other clinical signs. Hence, not all items/variables were available for all the individual patients and the final assessment of the signs depended on the individual level of experience and skills of the examiner.

Furthermore, we cannot rule out that the main outcome measure of this study - an ABL usually defined by a DWI lesion on delayed MRI - may not have missed a few ischemic strokes that were small enough to be MRI negative [7]. However, due to the retrospective design of the study under real life but uncontrolled circumstances, we regarded a DWI lesion on delayed MR imaging to be a more reliable marker for the final diagnosis of a stroke than the clinical diagnosis that may have been solely based on the clinical assessment (e.g., the HINTS examination) performed by different clinicians with varying levels of experience.

\section{Conclusion}

Patients presenting with dizziness, vertigo or imbalance to the ED should first be screened for a general medical cause $[8,30]$ as well as BPPV as a common and easily treatable disorder. Next, thorough history taking should include timing and triggers of dizziness symptoms and clinical examination should focus on central oculomotor signs and focal abnormalities indicating CNS involvement. Patients with any of the latter signs should undergo brain imaging, usually an early CT in the ED and a delayed MRI after admission. In the remaining patients, decision-making on the need, modality and timing of imaging studies can by guided by our proposed clinical pathway which integrates the empiric risk for ABLs into established clinical algorithms for dizzy patients. Using this practical approach could prevent many unnecessary brain imaging studies in dizzy patients without a central cause and assure imaging in those who require it.

Acknowledgments Open Access funding provided by Projekt DEAL.

Funding This work was supported by the German Research Foundation (Deutsche Forschungsgemeinschaft; Grant MA5332/3-1 to BM).

Availability of data and material The data that support the findings of this study are available from the corresponding author upon reasonable request.

\section{Compliance with ethical standards}

Conflicts of interest The authors declare that they have no conflicts of interest.

Ethics approval The study was approved by the Ethics Committee of the University of Lübeck (18-146A) and has therefore been performed in accordance with the ethical standards laid down in the 1964 Declaration of Helsinki and its later amendments.

Open Access This article is licensed under a Creative Commons Attribution 4.0 International License, which permits use, sharing, adaptation, distribution and reproduction in any medium or format, as long as you give appropriate credit to the original author(s) and the source, provide a link to the Creative Commons licence, and indicate if changes were made. The images or other third party material in this article are included in the article's Creative Commons licence, unless indicated otherwise in a credit line to the material. If material is not included in the article's Creative Commons licence and your intended use is not permitted by statutory regulation or exceeds the permitted use, you will need to obtain permission directly from the copyright holder. To view a copy of this licence, visit http://creativecommons.org/licenses/by/4.0/.

\section{References}

1. Brazzelli M, Chappell FM, Miranda H, Shuler K, Dennis M, Sandercock PA, Muir K, Wardlaw JM (2014) Diffusion-weighted imaging and diagnosis of transient ischemic attack. Ann Neurol 75:67-76

2. Chalela JA, Kidwell CS, Nentwich LM, Luby M, Butman JA, Demchuk AM, Hill MD, Patronas N, Latour L, Warach S (2007) Magnetic resonance imaging and computed tomography in emergency assessment of patients with suspected acute stroke: a prospective comparison. Lancet 369:293-298

3. Choi JH, Park MG, Choi SY, Park KP, Baik SK, Kim JS, Choi KD (2017) Acute transient vestibular syndrome: prevalence of stroke and efficacy of bedside evaluation. Stroke 48:556-562

4. Choi SY, Kee HJ, Park JH, Kim HJ, Kim JS (2014) Combined peripheral and central vestibulopathy. J Vestib Res 24:443-451

5. Cnyrim CD, Newman-Toker D, Karch C, Brandt T, Strupp M (2008) Bedside differentiation of vestibular neuritis from central "vestibular pseudoneuritis". J Neurol Neurosurg Psychiatr 79:458-460

6. Eagles D, Stiell IG, Clement CM, Brehaut J, Taljaard M, Kelly AM, Mason S, Kellermann A, Perry JJ (2008) International survey of emergency physicians' awareness and use of the Canadian Cervical-Spine Rule and the Canadian Computed Tomography Head Rule. Acad Emerg Med 15:1256-1261 
7. Edlow BL, Hurwitz S, Edlow JA (2017) Diagnosis of DWInegative acute ischemic stroke: a meta-analysis. Neurology $89: 256-262$

8. Edlow JA, Gurley KL, Newman-Toker DE (2018) A new diagnostic approach to the adult patient with acute dizziness. J Emerg Med 54:469-483

9. Gottesman RF, Sharma P, Robinson KA, Arnan M, Tsui M, Ladha K, Newman-Toker DE (2012) Clinical characteristics of symptomatic vertebral artery dissection: a systematic review. Neurologist $18: 245-254$

10. Grewal K, Austin PC, Kapral MK, Lu H, Atzema CL (2015) Missed strokes using computed tomography imaging in patients with vertigo: population-based cohort study. Stroke 46:108-113

11. Helmchen C, Machner B, Lehnen N, Jahn K, Schneider E, Sprenger A (2014) Current state of diagnostic management of acute vertigo: a survey of neurologists in Germany. J Neurol 261:1638-1640

12. Johnston SC, Rothwell PM, Nguyen-Huynh MN, Giles MF, Elkins JS, Bernstein AL, Sidney S (2007) Validation and refinement of scores to predict very early stroke risk after transient ischaemic attack. Lancet 369:283-292

13. Kattah JC (2019) Neuroimaging of the acute vestibular syndrome and vascular vertigo. In: Shaikh A, Ghasia F (eds) Advances in translational neuroscience of eye movement disorders. Springer International Publishing, Cham, pp 309-335

14. Kattah JC, Talkad AV, Wang DZ, Hsieh YH, Newman-Toker DE (2009) HINTS to diagnose stroke in the acute vestibular syndrome: three-step bedside oculomotor examination more sensitive than early MRI diffusion-weighted imaging. Stroke 40:3504-3510

15. Kerber KA, Brown DL, Lisabeth LD, Smith MA, Morgenstern LB (2006) Stroke among patients with dizziness, vertigo, and imbalance in the emergency department: a population-based study. Stroke 37:2484-2487

16. Kerber KA, Meurer WJ, Brown DL, Burke JF, Hofer TP, Tsodikov A, Hoeffner EG, Fendrick AM, Adelman EE, Morgenstern LB (2015) Stroke risk stratification in acute dizziness presentations: a prospective imaging-based study. Neurology 85:1869-1878

17. Kerber KA, Meurer WJ, West BT, Fendrick AM (2008) Dizziness presentations in US emergency departments, 1995-2004. Acad Emerg Med 15:744-750

18. Kerber KA, Morgenstern LB, Meurer WJ, McLaughlin T, Hall PA, Forman J, Fendrick AM, Newman-Toker DE (2011) Nystagmus assessments documented by emergency physicians in acute dizziness presentations: a target for decision support? Acad Emerg Med 18:619-626

19. Kerber KA, Newman-Toker DE (2015) Misdiagnosing dizzy patients: common pitfalls in clinical practice. Neurol Clin 33:565-575

20. Kim AS, Sidney S, Klingman JG, Johnston SC (2012) Practice variation in neuroimaging to evaluate dizziness in the $\mathrm{ED}$. Am J Emerg Med 30:665-672

21. Lawhn-Heath C, Buckle C, Christoforidis G, Straus C (2013) Utility of head CT in the evaluation of vertigo/dizziness in the emergency department. Emerg Radiol 20:45-49

22. Lee SH, Kim JS (2015) Acute diagnosis and management of stroke presenting dizziness or vertigo. Neurol Clin 33:687-698
23. Machner B, Gottschalk S, Sander T, Helmchen C, Rambold H (2007) Intralabyrinthine schwannoma affecting the low but not high frequency function of the vestibulo-ocular reflex: implications for the clinical diagnosis of chronic peripheral vestibular deficits. J Neurol Neurosurg Psychiatr 78:772-774

24. Makin SD, Doubal FN, Dennis MS, Wardlaw JM (2015) Clinically confirmed stroke with negative diffusion-weighted imaging magnetic resonance imaging: longitudinal study of clinical outcomes, stroke recurrence, and systematic review. Stroke 46:3142-3148

25. Navi BB, Kamel H, Shah MP, Grossman AW, Wong C, Poisson SN, Whetstone WD, Josephson SA, Johnston SC, Kim AS (2012) Application of the $\mathrm{ABCD}^{2}$ score to identify cerebrovascular causes of dizziness in the emergency department. Stroke 43:1484-1489

26. Navi BB, Kamel H, Shah MP, Grossman AW, Wong C, Poisson SN, Whetstone WD, Josephson SA, Johnston SC, Kim AS (2012) Rate and predictors of serious neurologic causes of dizziness in the emergency department. Mayo Clin Proc 87:1080-1088

27. Navi BB, Kamel H, Shah MP, Grossman AW, Wong C, Poisson SN, Whetstone WD, Josephson SA, Johnston SC, Kim AS (2013) The use of neuroimaging studies and neurological consultation to evaluate dizzy patients in the emergency department. Neurohospitalist 3:7-14

28. Newman-Toker DE (2016) Missed stroke in acute vertigo and dizziness: it is time for action, not debate. Ann Neurol 79:27-31

29. Newman-Toker DE, Della Santina CC, Blitz AM (2016) Vertigo and hearing loss. Handb Clin Neurol 136:905-921

30. Newman-Toker DE, Hsieh YH, Camargo CA Jr, Pelletier AJ, Butchy GT, Edlow JA (2008) Spectrum of dizziness visits to US emergency departments: cross-sectional analysis from a nationally representative sample. Mayo Clin Proc 83:765-775

31. Newman-Toker DE, Kattah JC, Alvernia JE, Wang DZ (2008) Normal head impulse test differentiates acute cerebellar strokes from vestibular neuritis. Neurology 70:2378-2385

32. Newman-Toker DE, Kerber KA, Hsieh YH, Pula JH, Omron R, Saber Tehrani AS, Mantokoudis G, Hanley DF, Zee DS, Kattah JC (2013) HINTS outperforms $\mathrm{ABCD}^{2}$ to screen for stroke in acute continuous vertigo and dizziness. Acad Emerg Med 20:986-996

33. Saber Tehrani AS, Kattah JC, Kerber KA, Gold DR, Zee DS, Urrutia VC, Newman-Toker DE (2018) Diagnosing stroke in acute dizziness and vertigo: pitfalls and pearls. Stroke 49:788-795

34. Saber Tehrani AS, Kattah JC, Mantokoudis G, Pula JH, Nair D, Blitz A, Ying S, Hanley DF, Zee DS, Newman-Toker DE (2014) Small strokes causing severe vertigo: frequency of false-negative MRIs and nonlacunar mechanisms. Neurology 83:169-173

35. Strupp M, von Stuckrad-Barre S, Brandt T, Tonn JC (2013) Teaching neuroimages: compression of the eighth cranial nerve causes vestibular paroxysmia. Neurology 80:e77

36. Tarnutzer AA, Lee SH, Robinson KA, Wang Z, Edlow JA, Newman-Toker DE (2017) ED misdiagnosis of cerebrovascular events in the era of modern neuroimaging: a meta-analysis. Neurology 88:1468-1477

37. Zwergal A, Mohwald K, Dieterich M (2017) Vertigo and dizziness in the emergency room. Nervenarzt 88:587-596 